\title{
Ganoderma Association with the Mortality of Acacia auriculiformis, Susceptibility to Different Hosts and Its Controls
}

\section{Mousumi Bhadra*}

Hyogo International House, B-404, 1-2-8 Wakinohama-Cho, Chu-Ku, Kobe-Shi, Hyogo-Ken, Japan

\begin{abstract}
An investigation was conducted to find out the association of Ganoderma spp. with the mortality of Acacia auriculiformis at Jahangirnagar University Campus, Bangladesh. Diseases severity of the tree was calculated where the highest $(52.2 \%)$ incidence was recorded in site-1 of the study area. Isolation and identification of the Ganoderma spp. and Trichoderma spp. were carried out. A total of 11 hosts were examined for examine the susceptibility of Ganoderma on host. All of the wild Ganoderma spp. viz., G. Iucidum-1, G. Iucidum-2, G. Iucidum-3 and G. applanatum preferred to use saw dust of Mangifera indica followed by Ceriops decandra whereas the least performance recorded in Albizia procera and Dipterocarpus turbinatus. Trichoderma isolates found effective to control Ganoderma infestation under field conditions.
\end{abstract}

Keywords: Ganoderma; Acacia auriculiformis; Trichoderma; Disease severity; Biological control

\section{Introduction}

The genus Ganoderma includes several wood decaying fungi on living trees as well as dead trunks and stumps, and has been recorded mostly in tropical and temperate countries. Generally, Ganoderma spp. cause extensive heart rots of standing trees by growing in the central, non-living woody tissues. Several studies have been carried out on Ganoderma diseases focusing on economic damage, severity of the disease and host range in many regions such as America, Asia, the Middle East and Europe [1]. Ganoderma lucidum has been reported as the causal organism of the heart rot disease of 91 hosts species Quercus spp. [2], Cocos nucifera [3], Camellia sinensis [4], Prunus persica [5], Vitis vinifera [6], Delonix regia and Cassia fistula. According to previous studies, several G. lucidum strains have been identified in the G. lucidum complex [7] having different host specificity. Control of root rot diseases is difficult as the pathogens survive on woody material in the soil. Green mould disease caused by Trichoderma spp. one of the serious problem of oyster mushroom and white button mushroom. It causes large economic losses to the mushroom growers. This was agreed by [8-11]. But this Trichoderma spp. has ability to control various plant diseases. This study was undertaken to examine the spread of root and stem rot disease in a particular study area, identify the causal agent of the disease and control the disease by means of biocontrol agent, Trichoderma. So, we can use this spent mushroom compost as a biofertilizer.

\section{Materials and Methods}

A study was carried out to calculate disease prevalence of Acacia auriculiformis at four selected different sites of Jahangirnagar University Campus, Bangladesh. Acacia auriculiformis are the dominant trees in every site of the campus. A total of fifty trees were randomly selected in each study area. Ganoderma spp. viz G. lucidum-1, G. lucidum-2, and G. lucidum-3 were collected from fully dead plants where as $G$. applanatum from partially dead trees. Symptomatology of infected trees due to Ganoderma was studied carefully.

\section{Identification of Ganoderma spp. and Trichoderma spp.}

In the present study, Ganoderma spp. was classified according to Corner [12] and Steyaert [13]. All of mycelium of four Ganoderma was hyaline, hyphae aseptate, basidiospores were thick walled, bitunicate, golden brown in color and ovate in shape. Colors of the colony of all wild Ganoderma were white (Figure 1) The morphological, microscopic and cultural characteristic features of Ganoderma lucidum found more or less similar with previous researchers $[1,14]$. There was no chlamydospore found during present study as described by previous workers.

\section{Disease severity index}

The trees were scored for disease classes on a scale of 0 to 4 (Table 1). The Disease Severity Index (DSI) was calculated using a modified method of Abdullah et al. [15] and Ilias [16].

\section{Isolation of wild Ganoderma spp.}

Both the pathogens and infested wood chips were cultured on PDA medium. Pieces $(1 \mathrm{~cm} \times 1 \mathrm{~cm})$ of pileus and wood chips $(1 \mathrm{~cm} \times 1$ $\mathrm{cm}$ ) were placed at the center of the plate separately. Three replications for each isolate were maintained and incubated at $32 \pm 2^{\circ} \mathrm{C}$. All of the isolates were pure cultured on PDA plates and stored at $4^{\circ} \mathrm{C}$ until further use.

\section{Morphological and cultural characteristics}

Morphological Characterization of Ganoderma such as shape, size, thickness, margin, color, texture of pileus was examined. Microscopic studies were done by stereoscopic binocular microscope (OLYMPUS SZ 61, magnification 40X with Camera DP20, Japan). Hyphal features, colony characteristics were recorded.

\section{Isolation of antagonist}

A total of four species of Trichoderma i.e., Trichoderma harzianum,

*Corresponding author: Mousumi Bhadra, Hyogo International House, B-404 1-2-8 Wakinohama-Cho, Chu-Ku, Kobe-Shi, Hyogo-Ken, Japan, Tel: 0795-442043; E-mail: srmmm38mousumi@gmail.com

Received: July 21, 2014; Accepted October 28, 2014; Published October 31 2014

Citation: Bhadra M (2014) Ganoderma Association with the Mortality of Acacia auriculiformis, Susceptibility to Different Hosts and Its Controls. J Plant Pathol Microb 5: 238. doi:10.4172/2157-7471.1000238

Copyright: (c) 2014 Bhadra M. This is an open-access article distributed under the terms of the Creative Commons Attribution License, which permits unrestricted use, distribution, and reproduction in any medium, provided the original author and source are credited. 

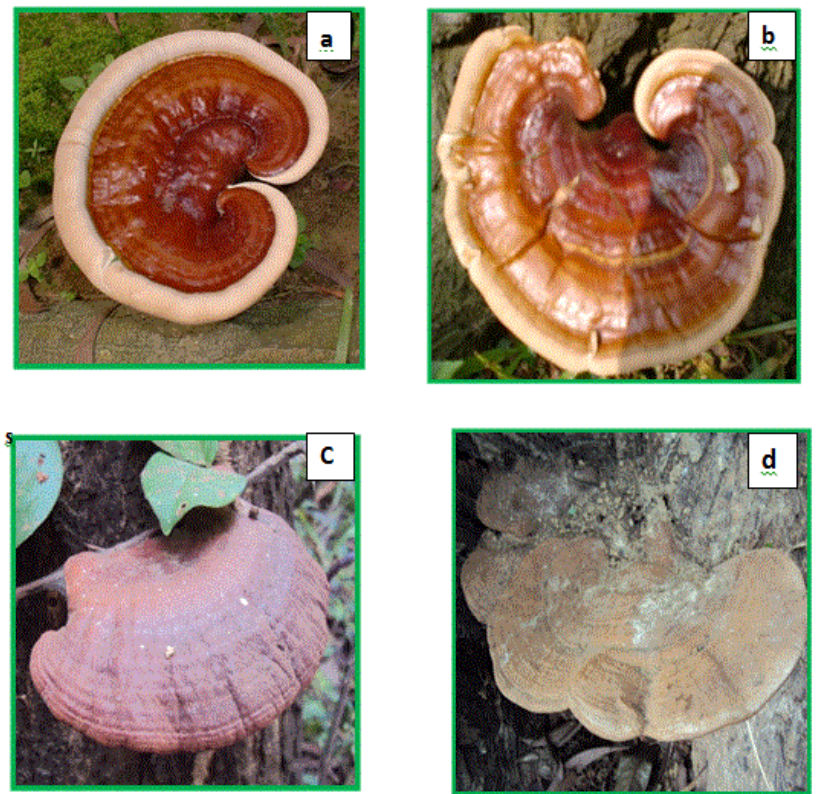

Figure 1: Photographs showing basidiocarps of studied Ganoderma spp.

a. Ganoderma lucidum 1 (wild)

b. Ganoderma lucidum 2 (wild)

c. Ganoderma lucidum 3 (wild)

d. Ganoderma applanatum (wild)

\begin{tabular}{|c|c|l|}
\hline $\begin{array}{c}\text { Disease } \\
\text { Classes }\end{array}$ & Range & Severity of infection \\
\hline $\mathbf{0}$ & 0 & Healthy plants \\
\hline $\mathbf{1}$ & $25>$ & Initial development of pinheads of Ganoderma spp. \\
\hline $\mathbf{2}$ & $26-50$ & $\begin{array}{l}\text { Appearance of Ganoderma on the tree trunk but no } \\
\text { remarkable damage observed in plants. }\end{array}$ \\
\hline $\mathbf{3}$ & $50-75$ & Partially top dying \\
\hline $\mathbf{4}$ & $>75$ & Fully top dying of plants and plants dried. \\
\hline
\end{tabular}

Formula used to calculate disease severity index $(D S I) \%=\Sigma(A \times B) \times 100 / \Sigma B \times 4$ where:

A - Disease classes $(0,1,2,3,4$ and so on)

$\mathrm{B}-$ Number of plants showing that disease classes per treatment

Table 1: Parameters used to calculate disease severity in the study area.

T. koningii, T. viride (green strain), and T. viride (yellow strain) were collected from infected spent mushroom spawn packets of Pleurotus ostreatus, during December'2010 to February'2011. Antagonists Trichoderma harzianum was characterized according to Barnett [17] and Choi et al. [18] and. Others strain of Trichoderma were characterized as described by Barnett [17].

\section{Assessment of Ganoderma susceptibility to different host range}

Sawdust of 11 randomly selected hosts viz., Acacia auriculiformis (L), Ceriops decandra (Griff.) Ding Hou, Tectona grandis L. f., Delonix regia (Bof.)Raf. Mangifera indica L., Dipterocarpus turbinatus Gaertn., Artocarpus chaplasha Roxb., Albizia procera Benth., Albizia lebbeck (L.) Benth., Artocarpus heterophyllus Lamk. were collected from Barisal Timbers \& Saw mills, Kazi Timbers \& Saw mills, M/S. N.S. Timbers etc. of Savar Bazar, Dhaka. Sawdusts were put in broad mouth test tubes $(20 \mathrm{~cm})$ and autoclaved $\left(121^{\circ} \mathrm{C}\right.$ temperature, 15 atm pressure for 20 minutes) for two times (a modified method of Fernando, 2008).
Four treatments combinations were used to assess susceptibility of Ganoderma. After cooling of saw dusts, fungal block each of $8 \mathrm{~mm}$ in size was inoculated into the test tubes containing sawdust under aseptic condition and cotton plugged. Test tubes were incubated at $32 \pm 2^{\circ} \mathrm{C}$ temperature. Radial growth of Ganoderma spp. on the test tube was measured at 10 days intervals and was analyzed statistically by MSTAT-C program.

Treatment $\mathrm{T}_{1}$ (Control) comprised of $70 \%$ Sawdust $+65 \%$ moisture+ inoculums (Ganoderma-1,2,3,4 separately); treatment $\mathrm{T}_{2}$ of $70 \%$ Sawdust $+65 \%$ moisture $+0.5 \% \mathrm{CaCO}_{3}+$ inoculums (Ganoderma-1,2,3,4 separately); thus Treatment $\mathrm{T}_{3}$ and $\mathrm{T}_{4}$ made up of $70 \%$ Sawdust $+65 \%$ moisture $+30 \%$ wheat bran $+2 \%$ sucrose $+0.5 \% \mathrm{CaCO}_{3}+$ inoculums (Ganoderma-1,2,3,4 separately) and 70\% Sawdust $+65 \%$ moisture $+30 \%$ rice bran $+2 \%$ sucrose $+0.5 \% \mathrm{CaCO}_{3}$ +inoculums (Ganoderma-1,2,3,4 separately) respectively.

\section{Field trial}

A field experiments were also conducted at Jahangirnagar University Campus during April to August 2011. A total of eight treatment combinations were used in field experiments (Table 2). A total of fifty non infected plants were randomly selected in these purpose to inoculate the isolated Ganoderma to examine the capacity to infest on living host and the control of diseases prevalence using antagonists.

\section{Mass culture of four Ganoderma isolates}

The bamboo chips $(3 \mathrm{~cm})$ were sun dried for 10 days after cutting. Then these chips were mixed with wheat bran $(30 \%)$, rice bean $(30 \%)$ and $2 \%$ sugar solution with maintained $65 \%$ moisture. Then, these substrates were put into broad mouth test tubes $(20 \mathrm{~cm})$ and plugged with cotton. These test tubes were autoclaved for two times and waited until cooled. Test tubes were inoculated with each of four Ganoderma species. Data was collected until test tubes were filled with Ganoderma mycelium.

\section{Preparation of Trichoderma spore suspension}

At first, $20 \mathrm{ml}$ of double distilled water was poured in each Petri dish having Trichoderma isolates. Then, each plate was scrapped to separate out mycelium of Trichoderma by using inoculating needle as mycelium of antagonist separated out. Then this solution was taken in plastic pot and covered with sterilized aluminum foil.

The stump or trunk region of Acacia sp. plants were holed by hammer and auger. Bamboo chips were inoculated into the plant by

\begin{tabular}{|c|l|}
\hline $\begin{array}{c}\text { Treatments } \\
\text { no. }\end{array}$ & Description \\
\hline Control & No inoculation of Ganoderma \\
\hline $\mathbf{T}_{1}$ & Inoculation of G-1. \\
\hline $\mathbf{T}_{\mathbf{2}}$ & Inoculation of G-2 \\
\hline $\mathbf{T}_{3}$ & Inoculation of $\mathrm{G}-3$ \\
\hline $\mathbf{T}_{\mathbf{4}}$ & Inoculation of $\mathrm{G}-4$ \\
\hline $\mathbf{T}_{\mathbf{5}}$ & Inoculation of $\mathrm{G}-1+$ addition of $T$. harzianum suspension \\
\hline $\mathbf{T}_{6}$ & Inoculation of $\mathrm{G}-2+$ addition of $T$. koningii suspension \\
\hline $\mathbf{T}_{\mathbf{7}}$ & Inoculation of $\mathrm{G}-3+$ addition of $T$. viride (green strain ) suspension \\
\hline $\mathbf{T}_{8}$ & Inoculation of G-3+addition of $T$. viride (yellow strain) suspension \\
\hline
\end{tabular}

Here, G1-Ganoderma lucidum-1, G2-Ganoderma lucidum-2, G3-Ganoderma lucidum-3, G4-Ganoderma applanatum.

$>5$ replication in each treatment except control where 10 replications were used.

Table 2: Treatments used to assesses the pathogenecity of Ganoderma spp. and its control using antagonistic potentiality of Trichoderma spp. at field condition. 
hammer and covered with adhesive tape. The mycelial run rate per bamboo chip was observed.

\section{Result and Discussion}

\section{Symptomatological study}

Ganoderma infected plants showed symptoms with initially a bleached zone appeared in the wood which results delignification, drying of apical meristem or top dying of plants was the common symptoms on plants, wilting of plants, stem blackening, defoliation, white rot and root rot, loss of stiffness and finally, death of tree plants. All of the Ganoderma infected plants consisted at least 5 to 7 fruit bodies except Ganoderma applanatum where infected plants contained one fruit body and plant was defoliated. The symptoms recorded during study are in conformity with some researchers $[14,19,20]$. Dysfunctional xylem associated with large wounds on the roots which were thought to be due to delignification; caused by Ganoderma applanatum [21].

\section{Calculation of Disease Severity Index (DSI)}

The DSI value of Ganoderma at four selected sites of Jahangirnagar University Campus revealed that the highest disease incidence was found in site-1 (55.2\%) in Acacia auriculiformis followed by site-2 (47.5\%), site-4 (45\%) and site-3 (36\%) (Table 3). Such findings partially supported by Nur and Abdullah [22] who cited the highest DSI (70.0\%) due to Ganoderma infected oil palm seedlings. Disease progression of basal stem rot symptoms caused by Ganoderma boninense was recorded $100 \%$ in oil palm seedlings [23].

\section{Assessment of wild Ganoderma (four) susceptibility to different host range}

Ganoderma lucidum-1 was susceptible Acacia auriculiformis due

\begin{tabular}{|c|c|c|c|c|c|}
\hline \multirow{2}{*}{ Disease Class } & \multirow{2}{*}{ Range } & \multicolumn{4}{|c|}{ No. of infected plants } \\
\cline { 3 - 6 } & & Site 1 & Site 2 & Site 3 & Site 4 \\
\hline $\mathbf{0}$ & 0 & 7 & 10 & 15 & 10 \\
\hline $\mathbf{1}$ & $25>$ & 10 & 10 & 13 & 10 \\
\hline $\mathbf{2}$ & $26-50$ & 8 & 10 & 12 & 15 \\
\hline $\mathbf{3}$ & $50-75$ & 15 & 15 & 5 & 10 \\
\hline $\mathbf{4}$ & $>75$ & 10 & 5 & 5 & 5 \\
\hline \multicolumn{2}{|c|}{ DSI (\%) } & 55.2 & 47.5 & 36 & 45 \\
\hline
\end{tabular}

Table 3: Disease severity index (DSI) of site -1, 2, 3, 4 at Jahangirnagar University Campus.

to treatment $\mathrm{T}_{2}$ which is statistically identical to $\mathrm{T}_{4}$ followed by $\mathrm{T}_{3}$ at 10 days (Table 4 ) but treatment $\mathrm{T}_{4}$ exhibited more susceptible at 30 days. More or less similar results found in case of Ganoderma lucidum-2 and Ganoderma lucidum-3. Ganoderma applanatum showed no significant differences at 10 days but at 20 and 30 days only saw dusts showed more susceptible. In case of Artocarpus chaplasha, Ganoderma lucidum-1 was susceptible to treatment $\mathrm{T}_{4}$ at 10,20,30 days respectively (Table 4). More or less similar patern recorded in case of Delonix regia (Table 5). Ganoderma applanatum showed better growth in sole saw dust of Delonix regia.

There was no clear pattern found in case of Albizia lebbeck for its growth (Table 4).

In case of Dipterocarpus turbinatus, Ganoderma lucidum-1 and 2 showed better growth in the treatment T4 during entire incubation period (Table 5). Ganoderma applanatum preferred to grow where $\mathrm{CaCO}_{3}$ was added up to 20 days but treatment $\mathrm{T}_{4}$ showed better performance at 30 days of inoculation. All of the Ganoderma spp. showed better growth performance in sole saw dust of Ceriops decandra (Table 5). In case of Artocarpus heterophyllus, Ganoderma lucidum-1 and 3 showed better performance in treatment $\mathrm{T}_{4}$ at

\begin{tabular}{|c|c|c|c|c|c|c|c|c|c|c|c|c|c|}
\hline & \multirow[b]{2}{*}{ Treatments } & \multicolumn{3}{|c|}{ Ganoderma lucidum 1} & \multicolumn{3}{|c|}{ Ganoderma lucidum 2} & \multicolumn{3}{|c|}{ Ganoderma lucidum 3} & \multicolumn{3}{|c|}{ Ganoderma applanatum } \\
\hline & & $\begin{array}{l}10 \text { days } \\
\text { (cm) }\end{array}$ & $\begin{array}{l}20 \text { days } \\
\text { (cm) }\end{array}$ & $\begin{array}{l}30 \text { days } \\
\text { (cm) }\end{array}$ & $\begin{array}{l}10 \text { days } \\
(\mathrm{cm})\end{array}$ & $\begin{array}{l}20 \text { days } \\
(\mathrm{cm})\end{array}$ & $\begin{array}{l}30 \text { days } \\
(\mathrm{cm})\end{array}$ & $\begin{array}{l}10 \text { days } \\
(\mathrm{cm})\end{array}$ & $\begin{array}{c}20 \text { days } \\
(\mathrm{cm})\end{array}$ & 30 days $(\mathrm{cm})$ & $\begin{array}{l}10 \text { days } \\
(\mathrm{cm})\end{array}$ & $\begin{array}{l}20 \text { days } \\
(\mathrm{cm})\end{array}$ & 30 days $(\mathrm{cm})$ \\
\hline \multirow{5}{*}{ 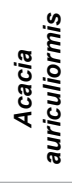 } & $\mathrm{T}_{1}$ (control) & $1.93 \mathrm{ab}$ & $6.0 \mathrm{~b}$ & $9.00 \mathrm{~d}$ & $2.00 \mathrm{c}$ & $6.20 \mathrm{c}$ & $9.60 \mathrm{c}$ & $2.70 \mathrm{~b}$ & $6.20 \mathrm{c}$ & $9.50 \mathrm{c}$ & $1.50 \mathrm{a}$ & $3.80 \mathrm{a}$ & $5.80 \mathrm{a}$ \\
\hline & $\mathrm{T}_{2}$ & $2.60 \mathrm{a}$ & $7.0 \mathrm{a}$ & $10.10 \mathrm{c}$ & $3.30 \mathrm{a}$ & $7.20 \mathrm{~b}$ & $11.27 \mathrm{~b}$ & $3.10 \mathrm{a}$ & $7.20 \mathrm{~b}$ & $11.00 \mathrm{~b}$ & $1.50 \mathrm{a}$ & $3.60 \mathrm{~b}$ & $5.20 \mathrm{~b}$ \\
\hline & $\mathrm{T}_{3}$ & $1.87 \mathrm{~b}$ & $6.20 \mathrm{ab}$ & $11.30 \mathrm{~b}$ & $3.03 \mathrm{~b}$ & $8.20 \mathrm{a}$ & $13.50 \mathrm{a}$ & $0.00 c$ & $0.00 \mathrm{~d}$ & $0.00 \mathrm{~d}$ & $1.40 \mathrm{a}$ & $3.20 \mathrm{c}$ & $0.00 \mathrm{c}$ \\
\hline & $\mathrm{T}_{4}$ & $2.57 \mathrm{a}$ & $6.20 \mathrm{ab}$ & $13.30 \mathrm{a}$ & $0.00 \mathrm{~d}$ & $0.00 \mathrm{~d}$ & $0.00 \mathrm{~d}$ & $2.70 \mathrm{~b}$ & $7.50 \mathrm{a}$ & $12.30 \mathrm{a}$ & $1.47 \mathrm{a}$ & $3.30 \mathrm{c}$ & $5.13 \mathrm{~b}$ \\
\hline & CV (\%) & 14.94 & 7.39 & 3.96 & 5.54 & 2.07 & 1.54 & 6.51 & 2.28 & 1.17 & 8.80 & 2.88 & 2.48 \\
\hline \multirow{5}{*}{ 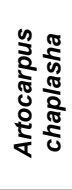 } & $\mathrm{T}_{1}$ (control) & $1.40 \mathrm{~b}$ & $2.80 \mathrm{~b}$ & $4.20 \mathrm{~b}$ & $0.00 \mathrm{c}$ & $0.00 \mathrm{c}$ & $0.00 \mathrm{c}$ & $2.00 \mathrm{~b}$ & $4.00 \mathrm{~b}$ & $6.00 \mathrm{~b}$ & $1.40 \mathrm{~b}$ & $2.80 \mathrm{~b}$ & $4.20 \mathrm{~b}$ \\
\hline & $\mathrm{T}_{2}$ & $1.67 \mathrm{~b}$ & $2.37 \mathrm{c}$ & $3.57 \mathrm{c}$ & $1.60 \mathrm{~b}$ & $3.20 \mathrm{~b}$ & $4.80 \mathrm{~b}$ & $2.00 \mathrm{~b}$ & $4.00 \mathrm{~b}$ & $6.00 \mathrm{~b}$ & $1.40 \mathrm{~b}$ & $2.80 \mathrm{~b}$ & $4.20 \mathrm{~b}$ \\
\hline & $\mathrm{T}_{3}$ & $1.40 \mathrm{~b}$ & $2.80 \mathrm{~b}$ & $4.20 \mathrm{~b}$ & $2.00 \mathrm{a}$ & $3.97 \mathrm{a}$ & $6.00 \mathrm{a}$ & $3.00 \mathrm{a}$ & $6.00 \mathrm{a}$ & $9.00 \mathrm{a}$ & $2.17 \mathrm{a}$ & $4.367 \mathrm{a}$ & $6.57 \mathrm{a}$ \\
\hline & $\mathrm{T}_{4}$ & $2.20 \mathrm{a}$ & $4.40 \mathrm{a}$ & $6.60 \mathrm{a}$ & $1.57 \mathrm{~b}$ & $3.17 \mathrm{~b}$ & $4.77 \mathrm{~b}$ & $3.00 \mathrm{a}$ & $6.00 \mathrm{a}$ & $9.00 \mathrm{a}$ & $1.40 \mathrm{~b}$ & $2.80 \mathrm{~b}$ & $4.20 \mathrm{~b}$ \\
\hline & CV (\%) & 7.72 & 3.85 & 2.56 & 5.00 & 2.58 & 1.66 & 4.00 & 2.00 & 1.33 & 6.02 & 3.00 & 2.00 \\
\hline \multirow{5}{*}{$\begin{array}{l}\frac{\pi}{3} \\
\frac{0}{0} \\
\frac{x}{0} \\
\frac{0}{0}\end{array}$} & $\mathrm{~T}_{1}$ (control) & $1.87 \mathrm{~b}$ & $5.87 \mathrm{c}$ & $8.87 \mathrm{~d}$ & $1.97 \mathrm{c}$ & $6.10 \mathrm{c}$ & $9.50 \mathrm{c}$ & $2.60 \mathrm{~b}$ & $6.10 \mathrm{c}$ & $9.47 \mathrm{c}$ & $1.42 a$ & $2.43 a$ & $5.70 \mathrm{a}$ \\
\hline & $\mathrm{T}_{2}$ & $2.60 \mathrm{a}$ & $6.87 \mathrm{a}$ & $10.27 \mathrm{c}$ & $3.20 \mathrm{a}$ & $7.10 \mathrm{~b}$ & $11.17 \mathrm{~b}$ & $2.97 \mathrm{a}$ & $7.07 \mathrm{~b}$ & $10.90 \mathrm{~b}$ & $1.05 b$ & $2.07 \mathrm{c}$ & $5.10 \mathrm{~b}$ \\
\hline & $\mathrm{T}_{3}$ & $2.60 \mathrm{a}$ & $6.10 \mathrm{~b}$ & $11.20 \mathrm{~b}$ & $2.87 \mathrm{~b}$ & $8.10 \mathrm{a}$ & $13.57 \mathrm{a}$ & $0.00 \mathrm{c}$ & $0.00 \mathrm{~d}$ & $0.00 \mathrm{~d}$ & $1.00 \mathrm{~b}$ & $2.10 \mathrm{~b}$ & $4.70 \mathrm{c}$ \\
\hline & $\mathrm{T}_{4}$ & $2.56 \mathrm{a}$ & $6.10 \mathrm{~b}$ & $13.20 \mathrm{a}$ & $0.00 \mathrm{~d}$ & $0.00 \mathrm{~d}$ & $0.00 \mathrm{~d}$ & $2.60 \mathrm{~b}$ & $7.40 \mathrm{a}$ & $12.20 \mathrm{a}$ & $1.00 \mathrm{~b}$ & $2.30 \mathrm{~b}$ & $4.97 \mathrm{~b}$ \\
\hline & CV (\%) & 3.66 & 1.34 & 3.05 & 3.62 & 0.94 & 1.28 & 5.48 & 0.97 & 0.94 & 1.01 & 1.15 & 1.49 \\
\hline \multirow{5}{*}{ 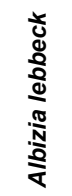 } & $\mathrm{T}_{1}$ (control) & $1.80 \mathrm{~b}$ & $5.60 \mathrm{c}$ & $8.90 \mathrm{~d}$ & $1.87 \mathrm{~d}$ & $5.87 \mathrm{~d}$ & $9.47 \mathrm{c}$ & $2.50 \mathrm{~b}$ & $5.20 \mathrm{c}$ & $9.30 \mathrm{c}$ & $1.40 \mathrm{a}$ & $4.10 \mathrm{a}$ & $5.60 \mathrm{a}$ \\
\hline & $\mathrm{T}_{2}$ & $2.50 \mathrm{a}$ & $6.70 \mathrm{a}$ & $9.77 \mathrm{c}$ & $3.06 \mathrm{a}$ & $6.67 \mathrm{~b}$ & $10.87 \mathrm{~b}$ & $2.97 \mathrm{a}$ & $6.77 \mathrm{~b}$ & $10.87 \mathrm{~b}$ & $1.30 \mathrm{~b}$ & $3.20 \mathrm{~b}$ & $4.97 \mathrm{~b}$ \\
\hline & $\mathrm{T}_{3}$ & $1.87 \mathrm{~b}$ & $4.77 \mathrm{~d}$ & $10.07 \mathrm{~b}$ & $2.80 \mathrm{~b}$ & $7.90 \mathrm{a}$ & $13.50 \mathrm{a}$ & $0.00 \mathrm{c}$ & $0.00 \mathrm{~d}$ & $0.00 \mathrm{~d}$ & $1.17 \mathrm{c}$ & $2.97 \mathrm{~d}$ & $2.97 \mathrm{c}$ \\
\hline & $\mathrm{T}_{4}$ & $2.40 \mathrm{a}$ & $5.80 \mathrm{~b}$ & $13.10 \mathrm{a}$ & $2.10 \mathrm{c}$ & $6.10 \mathrm{c}$ & $9.20 \mathrm{~d}$ & $2.50 \mathrm{~b}$ & $7.30 \mathrm{a}$ & $12.10 \mathrm{a}$ & $1.20 \mathrm{c}$ & $3.10 \mathrm{c}$ & $5.00 \mathrm{~b}$ \\
\hline & CV $(\%)$ & 3.57 & 1.34 & 0.80 & 3.59 & 1.33 & 0.82 & 3.83 & 1.59 & 0.95 & 2.28 & 0.86 & 0.72 \\
\hline
\end{tabular}

$\checkmark$ Means in a column followed by the same letter do not differ significantly at $5 \%$ level of significance (DMRT)

$\checkmark$ Treatment 1 (Control) $=$ only saw dusts + inocula (Ganoderma-1,2,3,4 separately), Treatment $2\left(\mathrm{~T}_{2}\right)=70 \%$ Sawdust $+65 \%$ moisture $+0.5 \%$ CaCO + inocula (Ganoderma-1,2,3,4 separately), Treatment $3\left(\mathrm{~T}_{3}\right)=70 \%$ Sawdust $+65 \%$ moisture $+30 \%$ wheat bran $+2 \%$ sucrose $+0.5 \%$ CaCO + inocula $($ Ganoderma- $1,2,3,4$ separately), Treatment $4\left(\mathrm{~T}_{4}\right)=70 \%$ Sawdust $+65 \%$ moisture $+30 \%$ rice bran $+2 \%$ sucrose $+0.5 \% \mathrm{CaCO}_{3}+$ inocula $($ Ganoderma- $1,2,3,4$ separately).

Table 4: Susceptibility of Ganoderma spp. to Acacia auriculiormis, Artocarpus chaplasha, Delonix regia and Albizia lebbeck at different days of inoculation. 
Citation: Bhadra M (2014) Ganoderma Association with the Mortality of Acacia auriculiformis, Susceptibility to Different Hosts and Its Controls. J Plant Pathol Microb 5: 238. doi:10.4172/2157-7471.1000238

Page 4 of 6

\begin{tabular}{|c|c|c|c|c|c|c|c|c|c|c|c|c|c|}
\hline & \multirow[b]{2}{*}{ Treatments } & \multicolumn{3}{|c|}{ Ganoderma lucidum 1} & \multicolumn{3}{|c|}{ Ganoderma lucidum 2} & \multicolumn{3}{|c|}{ Ganoderma lucidum 3} & \multicolumn{3}{|c|}{ Ganoderma applanatum } \\
\hline & & $\begin{array}{l}10 \text { days } \\
(\mathrm{cm})\end{array}$ & $\begin{array}{l}20 \text { days } \\
\text { (cm) }\end{array}$ & $\begin{array}{l}30 \text { days } \\
(\mathrm{cm})\end{array}$ & $\begin{array}{l}10 \text { days } \\
\text { (cm) }\end{array}$ & $\begin{array}{l}20 \text { days } \\
\text { (cm) }\end{array}$ & $\begin{array}{l}30 \text { days } \\
\text { (cm) }\end{array}$ & $\begin{array}{l}10 \text { days } \\
\text { (cm) }\end{array}$ & $\begin{array}{l}20 \text { days } \\
\text { (cm) }\end{array}$ & $\begin{array}{l}30 \text { days } \\
\text { (cm) }\end{array}$ & $\begin{array}{l}10 \text { days } \\
\text { (cm) }\end{array}$ & $\begin{array}{l}20 \text { days } \\
\text { (cm) }\end{array}$ & $\begin{array}{l}30 \text { days } \\
\text { (cm) }\end{array}$ \\
\hline \multirow{5}{*}{ 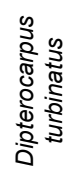 } & $\mathrm{T}_{1}($ control $)$ & $0.97 \mathrm{c}$ & $2.10 \mathrm{c}$ & $3.20 \mathrm{c}$ & $1.27 \mathrm{~b}$ & $1.27 \mathrm{~d}$ & $1.27 \mathrm{~d}$ & $1.30 \mathrm{~b}$ & $3.27 \mathrm{~b}$ & $4.87 \mathrm{~b}$ & $1.20 \mathrm{~b}$ & $2.70 \mathrm{c}$ & $4.10 \mathrm{~b}$ \\
\hline & $\mathrm{T}_{2}$ & $1.17 \mathrm{bc}$ & $2.40 \mathrm{~b}$ & $3.60 \mathrm{~b}$ & $1.20 \mathrm{~b}$ & $2.40 \mathrm{~b}$ & $3.60 \mathrm{~b}$ & $0.60 \mathrm{c}$ & $2.70 \mathrm{c}$ & $4.20 \mathrm{c}$ & $1.87 \mathrm{a}$ & $3.87 \mathrm{a}$ & $3.87 \mathrm{c}$ \\
\hline & $\mathrm{T}_{3}$ & $1.17 \mathrm{~b}$ & $1.67 \mathrm{~d}$ & $1.67 \mathrm{~d}$ & $1.87 \mathrm{a}$ & $1.87 \mathrm{c}$ & $1.87 \mathrm{c}$ & $2.20 \mathrm{a}$ & $4.40 \mathrm{a}$ & $6.60 \mathrm{a}$ & $1.00 \mathrm{c}$ & $2.10 \mathrm{~d}$ & $3.20 \mathrm{~d}$ \\
\hline & $\mathrm{T}_{4}$ & $1.30 \mathrm{a}$ & $3.80 \mathrm{a}$ & $6.50 \mathrm{a}$ & $1.77 \mathrm{a}$ & $4.10 \mathrm{a}$ & $7.60 \mathrm{a}$ & $0.00 \mathrm{~d}$ & $0.00 \mathrm{~d}$ & $0.00 \mathrm{~d}$ & $0.97 \mathrm{c}$ & $3.57 \mathrm{~b}$ & $5.67 \mathrm{a}$ \\
\hline & $\mathrm{CV}(\%)$ & 9.87 & 5.05 & 3.36 & 5.35 & 3.85 & 2.46 & 9.34 & 3.34 & 2.21 & 7.37 & 3.03 & 2.21 \\
\hline \multirow{5}{*}{ 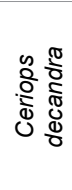 } & $\mathrm{T}_{1}($ control $)$ & $5.00 \mathrm{a}$ & $12.40 \mathrm{a}$ & $17.00 \mathrm{a}$ & $4.50 \mathrm{a}$ & $12.40 \mathrm{a}$ & $16.80 \mathrm{a}$ & $5.00 \mathrm{a}$ & $13.10 \mathrm{a}$ & $17.30 \mathrm{a}$ & $2.00 \mathrm{a}$ & $4.50 \mathrm{a}$ & $6.80 \mathrm{a}$ \\
\hline & $\mathrm{T}_{2}$ & $3.10 \mathrm{~b}$ & $10.57 \mathrm{~b}$ & $16.00 \mathrm{~b}$ & $1.80 \mathrm{~d}$ & $12.40 \mathrm{a}$ & $16.00 \mathrm{~b}$ & $4.70 \mathrm{a}$ & $11.80 \mathrm{~b}$ & $16.00 \mathrm{~b}$ & $1.80 \mathrm{~b}$ & $4.00 \mathrm{~b}$ & $6.30 \mathrm{~b}$ \\
\hline & $\mathrm{T}_{3}$ & $2.70 \mathrm{c}$ & $6.60 \mathrm{~d}$ & $11.30 \mathrm{~d}$ & $2.40 \mathrm{c}$ & $4.80 \mathrm{c}$ & $1.50 \mathrm{~d}$ & $3.20 \mathrm{~b}$ & $8.30 \mathrm{c}$ & $13.40 \mathrm{c}$ & $0.00 \mathrm{c}$ & $0.00 \mathrm{c}$ & $0.00 \mathrm{~d}$ \\
\hline & $\mathrm{T}_{4}$ & $3.20 \mathrm{~b}$ & $8.50 \mathrm{c}$ & $13.80 \mathrm{c}$ & $3.40 \mathrm{~b}$ & $9.00 \mathrm{~b}$ & $14.60 \mathrm{c}$ & $3.30 \mathrm{~b}$ & $8.20 \mathrm{c}$ & $13.60 \mathrm{c}$ & $1.90 \mathrm{ab}$ & $3.93 \mathrm{~b}$ & $5.10 \mathrm{c}$ \\
\hline & CV (\%) & 1.65 & 1.63 & 0.44 & 1.65 & 1.50 & 0.42 & 3.70 & 1.22 & 0.83 & 6.72 & 4.15 & 2.10 \\
\hline \multirow{5}{*}{ 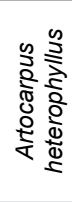 } & $\mathrm{T}_{1}$ (control) & $1.70 \mathrm{~b}$ & $3.40 \mathrm{~b}$ & $5.30 \mathrm{~b}$ & $0.00 \mathrm{c}$ & $0.00 \mathrm{c}$ & $0.00 \mathrm{c}$ & $2.27 b$ & $4.57 \mathrm{~b}$ & $7.17 \mathrm{~b}$ & $1.67 \mathrm{~b}$ & $3.37 \mathrm{~b}$ & $5.27 \mathrm{~b}$ \\
\hline & $\mathrm{T}_{2}$ & $1.50 \mathrm{c}$ & $2.97 \mathrm{c}$ & $4.70 \mathrm{c}$ & $1.80 \mathrm{~b}$ & $3.60 \mathrm{~b}$ & $5.70 \mathrm{~b}$ & $2.30 \mathrm{~b}$ & $4.60 \mathrm{~b}$ & $7.20 \mathrm{~b}$ & $1.70 \mathrm{~b}$ & $3.40 \mathrm{~b}$ & $5.30 \mathrm{~b}$ \\
\hline & $\mathrm{T}_{3}$ & $1.67 \mathrm{~b}$ & $3.37 \mathrm{~b}$ & $5.27 b$ & $2.30 \mathrm{a}$ & $4.60 \mathrm{a}$ & $7.20 \mathrm{a}$ & $3.17 \mathrm{a}$ & $6.37 \mathrm{a}$ & $9.80 \mathrm{a}$ & $2.50 \mathrm{a}$ & $5.00 \mathrm{a}$ & $7.70 \mathrm{a}$ \\
\hline & $\mathrm{T}_{4}$ & $2.50 \mathrm{a}$ & $5.00 \mathrm{a}$ & $7.70 \mathrm{a}$ & $1.80 \mathrm{~b}$ & $3.63 \mathrm{~b}$ & $5.70 \mathrm{~b}$ & $3.20 \mathrm{a}$ & $6.40 \mathrm{a}$ & $9.80 \mathrm{a}$ & $1.70 \mathrm{~b}$ & $3.40 \mathrm{~b}$ & $5.30 \mathrm{~b}$ \\
\hline & CV (\%) & 4.15 & 1.97 & 1.33 & 3.39 & 2.18 & 1.08 & 4.52 & 2.25 & 1.23 & 4.04 & 2.01 & 1.30 \\
\hline \multirow{5}{*}{ 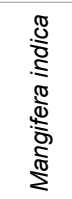 } & $\mathrm{T}_{1}$ (control) & $3.70 \mathrm{c}$ & $9.30 \mathrm{~d}$ & $16.00 \mathrm{a}$ & $3.20 \mathrm{~d}$ & $9.47 \mathrm{a}$ & $16.00 \mathrm{a}$ & $5.20 \mathrm{a}$ & $11.97 \mathrm{a}$ & $16.00 \mathrm{a}$ & $2.30 \mathrm{c}$ & $4.70 \mathrm{c}$ & $7.00 \mathrm{c}$ \\
\hline & $\mathrm{T}_{2}$ & $3.97 \mathrm{~b}$ & $11.60 \mathrm{~b}$ & $16.00 \mathrm{a}$ & $4.30 \mathrm{~b}$ & $11.80 \mathrm{a}$ & $16.00 \mathrm{a}$ & $4.50 \mathrm{~b}$ & $11.50 \mathrm{~b}$ & $16.00 \mathrm{a}$ & $2.30 \mathrm{c}$ & $5.20 \mathrm{~b}$ & $7.77 \mathrm{~b}$ \\
\hline & $\mathrm{T}_{3}$ & $4.20 \mathrm{a}$ & $10.10 \mathrm{c}$ & $16.00 \mathrm{a}$ & $5.27 \mathrm{a}$ & $43.17 \mathrm{a}$ & $16.00 \mathrm{a}$ & $4.97 \mathrm{a}$ & $10.97 \mathrm{c}$ & $16.00 \mathrm{a}$ & $4.50 \mathrm{a}$ & $9.50 \mathrm{a}$ & $16.00 \mathrm{a}$ \\
\hline & $\mathrm{T}_{4}$ & $3.80 \mathrm{c}$ & $12.50 \mathrm{a}$ & $16.00 \mathrm{a}$ & $4.07 \mathrm{c}$ & $11.57 \mathrm{a}$ & $16.00 \mathrm{a}$ & $3.77 \mathrm{c}$ & $9.867 \mathrm{~d}$ & $16.00 \mathrm{a}$ & $3.97 \mathrm{~b}$ & $9.20 \mathrm{a}$ & $16.00 \mathrm{a}$ \\
\hline & $\mathrm{CV}(\%)$ & 1.95 & 0.46 & 0.00 & 2.10 & 47.86 & 0.00 & 4.62 & 1.14 & 0.00 & 2.93 & 2.10 & 0.25 \\
\hline
\end{tabular}

$\checkmark$ Means in a column followed by the same letter do not differ significantly at $5 \%$ level of significance (DMRT)

$\checkmark$ Treatment 1 (Control) $=$ only saw dusts + inocula (Ganoderma-1,2,3,4 separately), Treatment $2\left(\mathrm{~T}_{2}\right)=70 \%$ Sawdust $+65 \%$ moisture $+0.5 \%$ CaCO ${ }_{3}+$ inocula (Ganoderma-1,2,3,4 separately), Treatment $3\left(\mathrm{~T}_{3}\right)=70 \%$ Sawdust $+65 \%$ moisture $+30 \%$ wheat bran $+2 \%$ sucrose $+0.5 \%$ CaCO + inocula $($ Ganoderma- $1,2,3,4$ separately), Treatment $4\left(\mathrm{~T}_{4}\right)=70 \%$ Sawdust $+65 \%$ moisture $+30 \%$ rice bran $+2 \%$ sucrose $+0.5 \% \mathrm{CaCO}_{3}+$ inocula $($ Ganoderma- $1,2,3,4$ separately).

Table 5: Susceptibility of Ganoderma spp. to Dipterocarpus turbinatus, Ceriops decandra, Artocarpus heterophyllus and Mangifera indica at different days of inoculation.

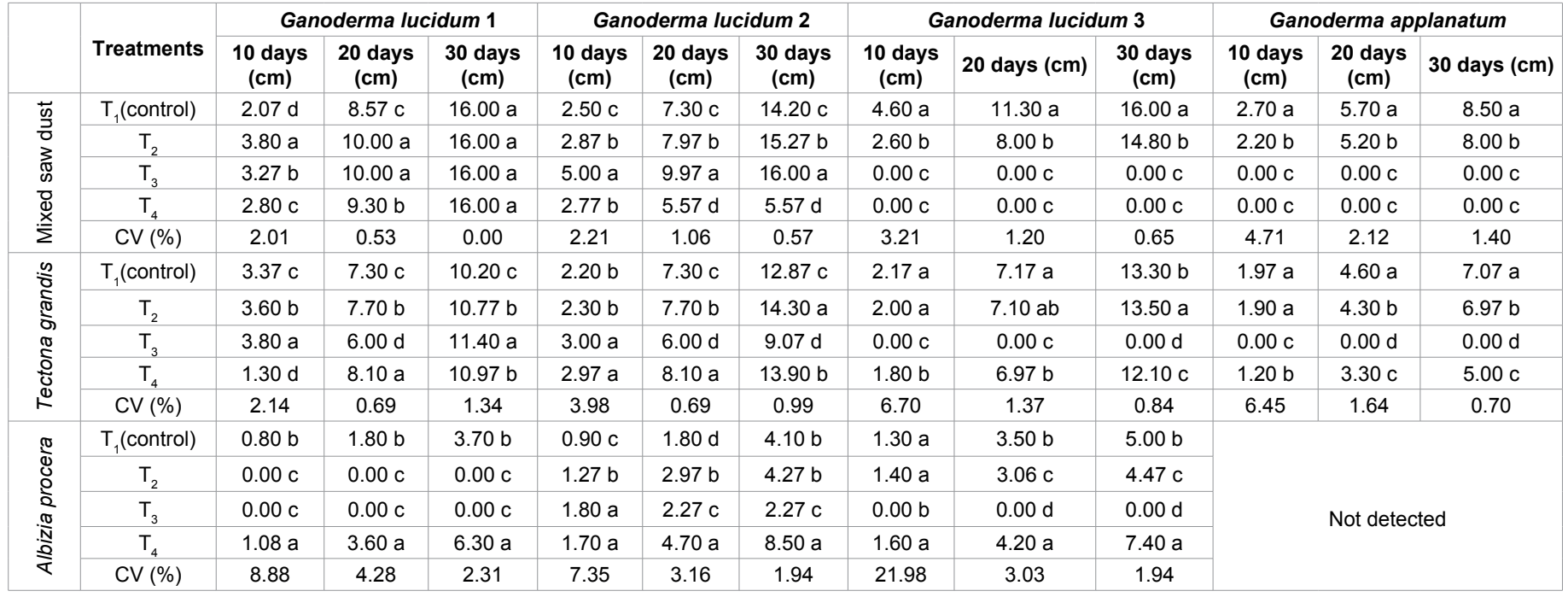

$\checkmark$ Means in a column followed by the same letter do not differ significantly at $5 \%$ level of significance (DMRT)

$\checkmark$ Treatment $1\left(\right.$ Control) $=$ only saw dusts + inocula (Ganoderma-1,2,3,4 separately), Treatment $2\left(\mathrm{~T}_{2}\right)=70 \%$ Sawdust $+65 \%$ moisture $+0.5 \%$ CaCO $3+$ inocula (Ganoderma-1,2,3,4 separately), Treatment $3\left(\mathrm{~T}_{3}\right)=70 \%$ Sawdust $+65 \%$ moisture $+30 \%$ wheat bran $+2 \%$ sucrose $+0.5 \% \mathrm{CaCO}_{3}+$ inocula $($ Ganoderma-1,2,3,4 separately), Treatment $4\left(\mathrm{~T}_{4}\right)=70 \%$ Sawdust $+65 \%$ moisture $+30 \%$ rice bran $+2 \%$ sucrose $+0.5 \% \mathrm{CaCO}_{3}+$ inocula $($ Ganoderma- $1,2,3,4$ separately)

Table 6: Susceptibility of Ganoderma spp. to mixed saw dust, Tectona grandi, and Albizia procera at different days of inoculation.

10, 20, 30 days respectively. Whereas, Ganoderma lucidum-2 and Ganoderma applanatum showed better growth in treatment $\mathrm{T}_{3}$ during entire period of study (Table 5). Ganoderma lucidum-1, 2, 3 showed better performances to all of the treatments at 30 days. Ganoderma applanatum preferred to use best performance in treatment $\mathrm{T}_{3}$ and $\mathrm{T}_{4}$ at 20, 30 days respectively (Table 5 ).
In case of mixed saw dust, Ganoderma lucidum-1 was susceptible to treatment $\mathrm{T}_{2}$ showed significant difference at 10 days but G. lucidum-1, 2 showed better performances in treatment $\mathrm{T}_{3}$ at 30 days. G. lucidum-3 and Ganoderma applanatum were prone to sole saw dust at 10, 20, 30 days respectively upto entire study (Table 6) G. lucidum-1 and 2 were commonly susceptible to Tectona grandis due to the treatment $\mathrm{T}_{3}$ at 
Citation: Bhadra M (2014) Ganoderma Association with the Mortality of Acacia auriculiformis, Susceptibility to Different Hosts and Its Controls. J Plant Pathol Microb 5: 238. doi:10.4172/2157-7471.1000238

Page 5 of 6

10 days and treatment $\mathrm{T}_{4}$ at 20 days. But at 30 days G. lucidum-1 and 2 showed better growth in treatment $\mathrm{T}_{3}$ and $\mathrm{T}_{2}$ respectively. G. lucidum-3 and $G$. applanatum showed more growth performance in treatment $\mathrm{T}_{2}$ at 30 days (Table 6). In case of $G$. lucidum-1, 2, 3 showed better performances in the treatment $\mathrm{T}_{4}$ at 30 days whereas G. applanatum showed no run rate in any treatments at any days (Table 6).

All of the Ganoderma spp. i.e., G. lucidum-1, G. lucidum-2, G. lucidum-3 and G. applanatum preferred to use saw dust of Mangifera indica followed by Ceriops decandra. This might be due to the presence of readily usable materials for its growth. In most of the cases, the least performance showed in Albizia procera and Dipterocarpus turbinatus might be the presence of secondary metabolites such as tannin, resins, and gums in wood. Root diseases caused by Ganoderma spp. seriously affected growth of Acacia spp. [24], Acacia auriculiformis and Acacia nilotica in India and Pakistan [25], Red rot disease of Acacia mangium caused by Ganoderma sp. reported in Malaysia [26]. Acacia auriculiformis affected by root rot disease caused by Ganoderma and
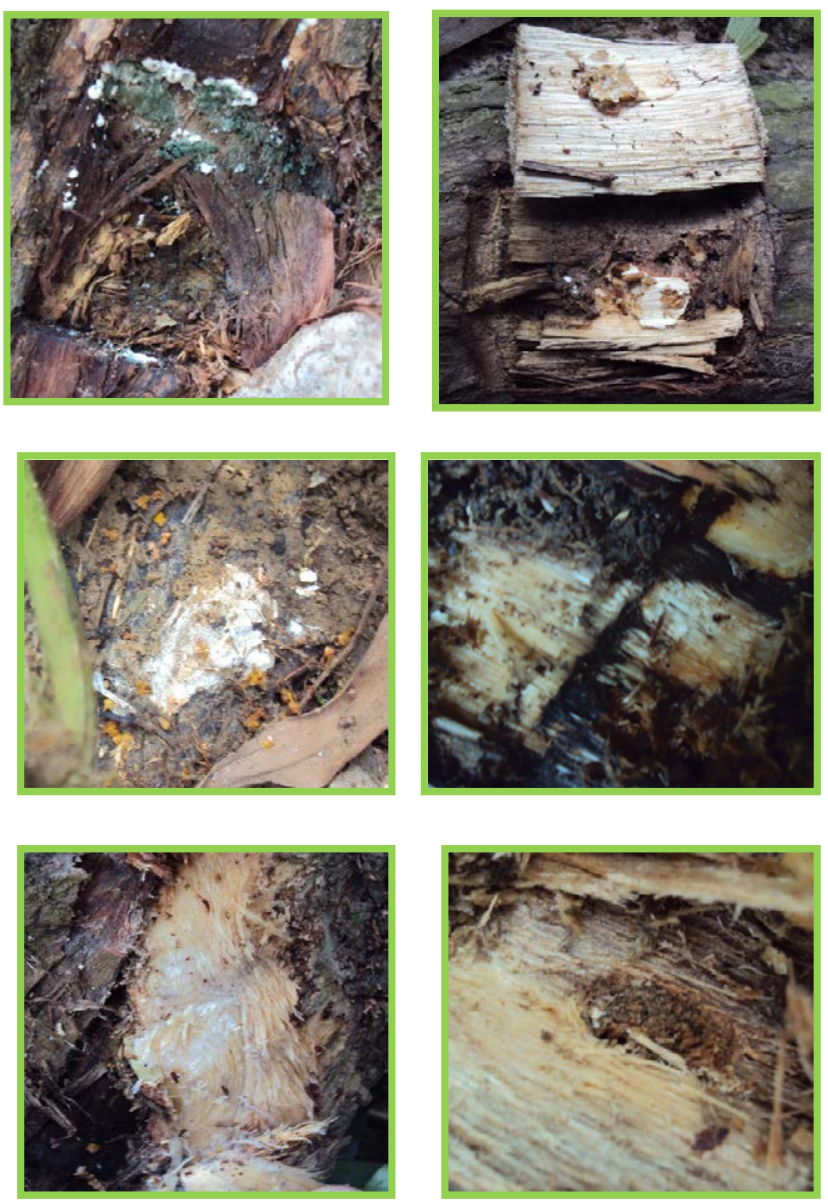

Figure 2: Photographs showing in vivo potentiality of Trichoderma and pathogenecity of Ganoderma spp.

a. Sporulation of Trichoderma over Ganoderma inoculated area

b. Colonization of Ganoderma mycelia

c. Colonization of Ganoderma lucidum-1 mycelia on the inoculated area d. Colonization of Ganoderma lucidum-2 mycelia on the inoculated area e. Colonization of Ganoderma lucidum-3 mycelia on the inoculated area f. Colonization of Ganoderma applanatum mycelia mycelia on the inoculated area (just initiated)
Phellinus spp. in Papua New Guinea [27], heart rot disease caused by Ganoderma lucidum recorded in Quercus spp., Cocos nucifera, Camellia sinensis, Prunus persica, Vitis vinifera, Cassia nodosa, Casia fistula, Delonix regia, others 144 hosts in India [1].

\section{Pathogenecity of Ganoderma spp. and antagonistic potentiality of Trichoderma spp.}

In vivo pathogenecity of four Ganoderma spp. showed colonization of mycelium within 5 months, that means trees inoculated with bamboo chips of Ganoderma lucidum-1, G. lucidum-2, G. lucidum-3, $G$. applanatum showed mycelial run rate in the surroundings of inoculated area. In case of Ganoderma applanatum, mycelial run rate was just initiated (Figure 2). In vivo evaluation of potentiality of Trichoderma against Ganoderma spp. also studied. Bamboo chips of four Ganoderma spp. were inoculated with four selected Trichoderma spp. showed no mycelial run rate of Ganoderma spp., even Trichoderma spp. was sporulated over the inoculated area. Ganoderma applanatum as the most degradative wood colonizer [14]. The pathogenicity of Ganoderma spp. and the inhibitory effect of Trichoderma on Ganoderma spp. was followed earlier research [28].

\section{Acknowledgement}

I am grateful to Professor Dr. Abul Khair, Professor Dr. Nazmul Alam and Professor Ali Akond Mamun for their critical reading and helpful comments. I also thank Mr. Saleh Ahmed Khan and Mr. Anawar Hossain from National Mushroom Development and Extension Centre, Savar, Dhaka for their technical assistance to undertake this study

\section{References}

1. Fernando KMEP (2008) The host preference of a Ganoderma lucidum strain for three tree species of Fabaceae family; Cassia nodosa, Cassia fistula and Delonix regia. Journal of the National Science Foundation of Sri Lanka. 36 323-326.

2. Grand LF, Jones RK (2001) Root and butt rot of oaks. Plant Pathology Extension, College of Agriculture and life Science, North Carolina State University.

3. Karthikeyan M, Radhika K, Bhaskaran R, Mathiyazhagan S, Samiyappan R et al. (2007) Pathogenicityearly diagnosis technique. Journal of Phytopathology. 155: 296-304.

4. Mauli C (1996) Diseases of tea

5. Advaskaveg JE and Gilbertson RL (1993) Wood decay, lignicolous and decline of peach trees in South Carolina. Plant Disease. 77: 707-711.

6. Advaskaveg JE, Gilbertson RL (1987) Infection and colonization of grapevines by Ganoderma lucidum. Plant Disease. 71: 251-253.

7. Miller RNG, Hoderness M, Bridge PD, Chung GF, Zakaria MH (1999) Genetic diversity of Ganoderma in oil palm plantings. Plant Pathology. 45: 595-603.

8. Castle A, Donna S, Nezar R, Glen A, Dan R et al. (1998) Morphological and Molecular Identification of Trichoderma Isolates on North American Mushroom Farms. Applied Environmental Microbiology. 64: 133-137.

9. Hatvani L, Antal Z, Manczinger L, Szekeres A, Druzhinina IS, et al. (2007) Green Mold Diseases of Agaricus and Pleurotus spp. Are Caused by Related but Phylogenetically Different Trichoderma Species. Phytopathology 97: 532537.

10. Monika KZ, Bissett J, Zafari D, Hatvani L, Manczinger L, et al (2007) Genetically Closely Related but Phenotypically Divergent Trichoderma Species Cause Green Mold Disease in Oyster Mushroom Farms Worldwide. Applied and Environmental Microbiology. 73: 7415-7426.

11. Savoie JM, lapicco R, Michele LLM (2001) Factors influencing the competitive saprophytic ability of Trichoderma harzianum Th2 in mushroom (Agaricus bisporus) compost. Mycological Research. 105: 1348-1356.

12. Corner EJH (1983) Ad Polyporaceas I. Amauroderma and Ganoderma. Nova Hedwigia 75: 1-182.

13. Steyaert RL (1972) Species of Ganoderma and related genera mainly of the Bogor and Lieden herbaria. Persoonia. 7: 55-118. 
Citation: Bhadra M (2014) Ganoderma Association with the Mortality of Acacia auriculiformis, Susceptibility to Different Hosts and Its Controls. J Plant Pathol Microb 5: 238. doi:10.4172/2157-7471.1000238

14. Schwarze FWMR, Ferner D (2003) Ganoderma on Trees-Differentiation of species and studies of invasiveness. ENSPEC. 27: 1-21.

15. Abdullah F, Ilias GNM, Nelson MMZ, Nur IA, Kalsom YU (2003) Disease assessment and the efficacy of Trichoderma as a biocontrol agent of basal stem rot of oil palms. Research Bulletin Scinence Putra. 11: 31-33.

16. Ilias GNM (2000) Trichoderma and its efficacy as a bio-control agent of basal stem rot of oil palm (Elaeis guineensis Jacq.). [Ph.D. Thesis.] University Putra Maᄀlaysia, Selangor, Malaysia.

17. Barnett HL (1960) Illustrated Genera of Imperfect Fungi. Second Edition. Burgees Pub. Co. Minneapoils, U.S.A.

18. Choi IY, Choi JN, Praveen KS, Lee WH (2003) Molecular and Morphological Characterization of Green Mold, Trichoderma spp. isolated from Oyster Mushrooms. The Korean Society of Mycology. 31: 74-80.

19. Pathak PS (1986) Mortality in leucaena due to Ganoderma lucidum. Leucaena Research Reports. 7: pp 65.

20. Schwarze FWMR, Baum S (2000) Mechanisms of reaction zone penetration by decay fungi in wood of beech (Fagus sylvatica). New Phytol. 146: 129-140.

21. Petersen JE (1983) Ganoderma in Northern Europe. Lakporesvampene (Ganoderma) i Danmark og Europa in Svampe. 7: 1-11.

22. Nur AIMZ, Abdullah F (2008) Disease suppression in Ganoderma-infected oil palm seedlings treated with Trichoderma harzianum. Plant Protection and Sciences. 44: 101-107.
23. Sundram S, Idris AS (2009) Trichoderma as a biocontrol agent against Ganoderma in oil palm. Malaysian Palm Oil Board. Ministry of Plantation Industries \& Commodities. Malaysia. MPOB Information Series. ISSN (463) $1511-7871$.

24. FAO (1981) Seed source establishment and tree improvement -Sabah, Malaysia. Forest Mycology. Rome, FAO/UNDP/MAL/78-009. p. 45.

25. Bagchee K (1945) Pathological notes: No. 2. Wilt and dieback on shisham (Dalbergia sissoo), babul (Acacia nilotica) and khair (A. catechu) in the artificial regeneration under agriculture-cum-forestry management. India Forester. 71 : $20-24$

26. Lee SS (1985) Tree diseases and wood deterioration problems in Peninsular Malaysia. Occasional Paper No. 5, Faculty of Forestry, Agriculture, University of Malaysia. pp 15.

27. Skelton DJ, Howcroft HS (1987) Seed production and silvicultural trials of Acacia in Papua New Guinea. In: Trurnbull, J.W. (ed.), Proceedings of an International Workshop, Australian Acacias in Developing Countries. Forestry Training Centre, Gympie, Queensland, Australia, August 1986. ACIAR Proceedings Series No. 16: 188-190.

28. Schwarze FWMR, Fink S (1994) Ermittlung der Holzzersetzung am lebenden Baum - Möglichkeiten und Grenzen verschiedener Diagnosemethoden zur Erfassung von Holzzersetzungsmustern. Neue Landschaft. 39:182-193. 